\title{
Performance of the AOAC use-dilution method with targeted modifications: Collaborative study
}

\section{Authors: Stephen F. Tomasino, Albert E. Parker, Martin A. Hamilton, \& Gordon C. Hamilton}

Notice: This is a postprint of an article that originally appeared in Journal of AOAC International on November 2012. DOI: http://dx.doi.org/10.5740/jaoacint.12-170.

Tomasino SF, Parker AE, Hamilton MA, Hamilton GC, "Performance of the AOAC use-dilution method with targeted modifications: Collaborative study," Journal of AOAC International. November 2012 95(6):1618-1628. 


\title{
Performance of the AOAC Use-Dilution Method with Targeted Modif cations: Collaborative Study
}

\author{
STEPHEN F. TOMASINO \\ Environmental Science Center, Fort Meade, MD 20755-5350 \\ ALBERT E. PARKER \\ Montana State University, Center for Biof lm Engineering, Bozeman, MT 59715 \\ MARTIN A. HAMILTON and GORDON C. HAMILTON \\ Big Sky Statistical Analysts LLC, Bozeman, MT 59715
}

U.S. Environmental Protection Agency, Off ce of Pesticide Programs, Biological and Economic Analysis Division, Microbiology Laboratory Branch,

The U.S. Environmental Protection Agency (EPA), in collaboration with an industry work group, spearheaded a collaborative study designed to further enhance the AOAC use-dilution method

(UDM). Based on feedback from laboratories that routinely conduct the UDM, improvements to the test culture preparation steps were prioritized. A set of modif cations, largely based on culturing the test microbes on agar as specif ed in the

AOAC hard surface carrier test method, were evaluated in a f ve-laboratory trial. The modif cati ons targeted the

preparation of the Pseudomonas aeruginosa test culture due to the diff culty in separating the pellicle from the broth in the current UDM. The proposed modif cations (i.e., the modif edUDM)werecomparedtothecurrentUDMmethodologyfor $P$. aeruginosa and Staphylococcus aureus. Salmonella choleraesuis was not included in the study. The goal was to determine if the modif cations reduced method variability. Three eff cacy response variables were statistically analyzed: the number of positive carriers, the log reduction, and the pass/fail outcome. The scope of the collaborative study was limited to testing one liquid disinfectant (an EPA-registered quaternary ammonium product) at two levels of presumed product eff cacies, high and low. Test conditions included use of $400 \mathrm{ppm}$ hard water as the product diluent and a $5 \%$ organic soil load (horse serum) added to the inoculum. Unfortunately, the study failed to support the adoption of the major modif cation (use of an

agar-based approach to grow the test cultures) based on an analysis of method's variability. The repeatability and reproducibility standard deviations for the modif ed method were equal to or greater than those for the current method across the various test variables. However, the authors propose retaining the frozen stock preparation step of the modif ed method, and based on the statistical equivalency of the control log densities, support its adoption as a procedural change

to the current UDM. The current UDM displayed acceptable responsiveness to changes in product efficacy; acceptable

repeatability across multiple tests in each laboratory for the control counts and log reductions; and acceptable reproducibility across multiple laboratories for the control log density values and log reductions. Although the data do not support the adoption of all modifications, the UDM collaborative study data are valuable for assessing sources of method variability and a reassessment of the performance standard for the UDM.

The U. S. Environmental Protection Agency (EPA) regulations specify that efificacy data must be submitted to support the registration of antimicrobial products bearing claims to control microorganisms on inanimate surfaces that pose a threat to human health. The registrant must ensure that the product is tested in accordance with the label directions. The AOAC use-dilution methods (UDMs; 1-3), 955.14 (Salmonella enterica), 955.15 (Staphylococcus aureus) and 964.02 (Pseudomonas aeruginosa), are standard methods used to substantiate eff cacy claims for liquid formulations. With the exception of the test microbe and the preparation of test culture, the three UDMs are essentially the same. Furthermore, to monitor product performance following registration, the EPA uses the UDMs where applicable in its post registration product stewardship program.

Historically, UDM has been the subject of criticism due to vagueness of the published standards and technique-sensitive aspects of the method, which have been cited as likely sources of variability in the eff cacy results. Two signif cant multilaboratory studies by Cole and Rutala (4) and Cole et al. (5) demonstrated the occurrence of variability among laboratories and identif ed major aspects of the method deemed as likely sources of the variability, most notably irregular surfaces of the stainless steel carriers (penicylinders), inconsistent inoculum levels, and inadequate removal of the pellicle associated with P. aeruginosa test cultures.

Standardization and monitoring of carrier loading were not addressed in those studies; this aspect may have contributed to variability in the eff cacy results. Furthermore, the misinterpretation and the subjective nature of the UDM probably have contributed to a wide range of working protocols, i.e., 
standard operating procedures (SOPs), making the comparison of multilaboratory data even more challenging.

An initiative designed to resolve concerns with the UDM led to the development and validation of AOAC Off cial Method ${ }^{\mathrm{SM}}$ 991.47, the hard surface carrier test (HSCT; 6, 7), which is also accepted by EPA to support the registration of liquid disinfectants. However, the HSCT has been used sparingly by the regulated community, purportedly due to the lack of hard water and organic soil load in the validation study.

Over the past several years, the EPA has led an effort to improve the UDM by using the off cial AOAC INTERNATIONAL method revision process. As a result, a revised version of the UDM was published in the AOAC Off cial Methods of Analysis in 2006. The editorial revisions focused on updating sources of reagents and supplies and increasing specif city of the procedures by providing concise, easy to follow steps without extensive cross-referencing. Recently, signif cant improvements to the UDM were made by introduction of a procedure to assess the number of viable cells per dried inoculated control carriers and a minimum carrier density (geometric mean density of $10^{6}$ across 6 control carriers) requirement for $S$. aureus and P. aeruginosa (8). The revisions were approved by AOAC in 2009 and are presently First Action status revisions. Recently, a procedural modif cation was approved by AOAC to establish a maximum geometric mean log density of $10^{7}$ per carrier for methods 955.15 and 964.02 (9). As a result of the control carrier count revisions, the UDM may be characterized as a semiquantitative procedure, rather than a strict qualitative one, thus allowing for additional statistical analyses and interpretation of results. In the current report, in addition to analyzing the number of positive carriers directly, the availability of the control counts allows the log reduction for each UDM test to be estimated directly, thereby determining the variability of the UDM with respect to log reductions. This calculation allows for a direct comparison of the variability observed for the UDM to quantitative tests published in the literature (10). Although this approach may be considered new for the analysis of semiquantitative eff cacy data, it has been implemented in previous studies of the UDM (11) and the HSCT (12).

EPA is pursuing the adoption of a quantitative eff cacy test method based on American Society for Testing and Materials method 2197-02 (13) to replace the UDM for measuring the performance of antimicrobial products. The methodology under consideration is the result of an international harmonization initiative under the purview of the Organization of Economic and Cooperative Development (OECD). Implementation of the new methodology, the OECD Quantitative Method for Evaluating Bactericidal Activity of Microbicides Used on Hard Non-Porous Surfaces, and the development of quantitative performance standards may require several years to complete. Thus, as an interim measure, efforts to enhance the performance of the UDM by introducing targeted procedural changes are justif ed and should be undertaken.

In collaboration with an industry-based technical work group, EPA submitted a survey to a wide range of laboratories that conduct the UDM to help identify and prioritize areas of different practices and interpretation of the procedure. Based on the feedback, methods for the preparation and harvest of the test cultures were determined to be the most divergent among laboratories. Thus, modif cations associated with the preparation and harvest of the test cultures were prioritized for this study.
Most importantly, the modif cations targeted the preparation of the $P$. aeruginosa test culture due to the diff culty in separating the pellicle from the broth. The potential effects of the pellicle have been documented previously $(14,15)$. Although the UDM method $\mathbf{9 6 4 . 0 2}$ provides specif $\mathrm{c}$ instructions for avoiding the disruption of the pellicle during the harvesting process, concerns were raised over the potential impact of microscopic pellicle fragments associated with f nal test cultures on the variability of product performance data. To provide the data necessary to support procedural changes and to assess improvement in method performance (i.e., reduction in method variability), a collaborative study was conducted to compare the results of the current version of the UDM to a modif ed version. The proposed modif cations were classif ed as procedural changes by AOAC, thus requiring a multilaboratory evaluation. The targeted modif cations were largely based on the HSCT methodology.

For this study, $S$. aureus and $P$. aeruginosa were prioritized due to their regulatory importance associated with hospital disinfectant claims. EPA's SOP for conducting the UDM, SOP MB-05 (16), is based on a strict interpretation of the published UDM, and was followed by the participating labs. The modif ed version of the UDM was the current method with two modif cations: (1) use of frozen stock cultures (stored at $-70^{\circ} \mathrm{C}$ or below) instead of stock cultures grown and stored on agar slants and stabs; and (2) the use of an agar-based procedure to grow the test microbes as a means to mitigate the impact of pellicle. The modif ed UDM included both modif cations; thus, the modif cations were not evaluated separately.

This paper reports the results of this collaborative study. This report includes comparisons of the two UDM methods with respect to the control counts of viable organisms on the inoculated carriers, the number of positive carriers out of 60 , and the log reductions. For each UDM method, the repeatability across tests in each laboratory and the reproducibility across laboratories are compared to results from other published disinfectant methods.

\section{Collaborative Study}

\section{Applicability}

The scope of this study was limited to testing a liquid disinfectant against $S$. aureus and $P$. aeruginosa on a hard, nonporous surface (stainless steel penicylinders). The generation of the product performance data in this study for an EPA-registered product was for research purposes only. Therefore, the product used in the study was an experimental component only and was not evaluated to support or verify product label claims. The test conditions included the use of $400 \mathrm{ppm}$ hard water as the product diluent and a $5 \%$ organic soil load (horse serum) added to the inoculum.

\section{Collaborators and Quality Management}

Five volunteer laboratories (denoted as A, B, C, D, and E) participated in this study: one federal laboratory; one contract laboratory; and three industry laboratories. The laboratories had existing microbiology programs, appropriately trained personnel, and had the capability of conducting the protocol within the established timeframe. The EPA Biological and Economic Analysis Division-Microbiology Laboratory Branch, 
Fort Meade, MD, facilitated the collaborative study and served as the lead laboratory. The participating laboratories had a history of conducting the UDM and were assumed to be representative of laboratories that routinely conduct the UDM. Analysts performing the tests were asked to familiarize themselves with each version of the UDM method and to build prof ciency by the use of in-house practice sessions. Following data collection, the data sheets were submitted in a blind-coded fashion to an industry liaison for initial peer review, and were then forwarded to the EPA study director, who performed a f nal peer review and submitted the data in spreadsheet format to the statisticians for statistical analysis. Thorough documentation was maintained to ensure that the study was supported by complete, accurate, and chronological records from initial collection of raw data to f nal analysis and reporting of results. The level of quality assurance was consistent with data collection principles specif ed under EPA Good Laboratory Practice Standards - FIFRA 40 CFR Part 160 (17).

\section{Study Design}

The study design was consistent with past AOAC-approved method modif cation and validation studies conducted by EPA; however, AOAC did not off cially facilitate the process. $S$. aureus (ATCC 6538) and P. aeruginosa (ATCC 15442) were the test microbes. EPA SOP MB-05, based on the 2009 revised AOAC Off cial Methods 955.15 and 964.02, was considered the current UDM. The current UDM and the modif ed UDM method (which included the two proposed modif cations) were conducted side-by-side on each test day so that the results could be compared directly.

One representative EPA-registered quaternary ammoniumbased product was used as the experimental test chemical. The product's label ingredients were: (1) $6.510 \%$ octyl decyl dimethyl ammonium chloride; (2) 3.255\% dioctyl dimethyl ammonium chloride; (3) 3.255\% didecyl dimethyl ammonium chloride; and (4) $8.860 \%$ alkyl $\left(50 \% \mathrm{C}_{14}, 40 \% \mathrm{C}_{12}\right.$, and $\left.10 \% \mathrm{C}_{16}\right)$ dimethyl benzyl ammonium chlorides. Screening studies were conducted prior to the off cial study to determine the product test conditions. The goal was to establish a range of product eff cacy to measure the responsiveness of the methods, an important method performance criterion. On each test day, a presumed high-eff cacy level of the product was tested using both versions of the UDM, followed by testing of a presumed low-eff cacy level of the product by both methods. Product dilution for the high-eff cacy level was based on the product's label claim (1:256) and was the same for both organisms; however, for the low-eff cacy level, dilutions of 1:576 and 1:1280 were used for $P$. aeruginosa and $S$. aureus, respectively. The higher product dilution for $S$. aureus was necessary due to the microbe's greater sensitivity to the product. The product label claims were used to determine the test conditions for the organic burden, diluent, contact time and temperature. The diluent was sterile $400 \mathrm{ppm}$ (as calcium carbonate) AOAC synthetic hard water prepared per AOAC Off cial Method $\mathbf{9 6 0 . 0 9}$ (18). Organic burden in the form of $5 \%(\mathrm{v} / \mathrm{v})$ horse serum was added to the inoculum prior to carrier inoculation.

The product was tested with a 10 min contact time at a temperature of $20 \pm 1^{\circ} \mathrm{C}$. The neutralizer for the product was conf rmed prior to use and consisted of letheen broth with $2 \times$ lecithin plus polysorbate 80 . A single tube of neutralizer/subculture medium was used per carrier. Physically and biologically screened carriers were utilized in the study. The product was distributed to the testing laboratories in containers that were marked only with a treatment code. A single lot of the test chemical was used. In advance of testing, an analysis of formulation chemistry was performed to conf rm the level of active ingredients. EPA chemists (Biological and Economic Analysis Division Analytical Chemistry Branch) were responsible for the formulation chemistry, sample labeling, and shipment.

The laboratories were instructed to perform the same number of tests/day: four 60 -carrier tests. The set of four 60 -carrier tests was replicated on three separate test days/microbe to provide data for an assessment of within-laboratory variability. The order of testing against $S$. aureus and P. aeruginosa was randomized for each laboratory; however, the high-eff cacy treatment was always performed $\mathrm{f}$ rst on each test day to mitigate cross-contamination to the low-eff cacy test carriers. Therefore, each of the $\mathrm{f}$ ve laboratories conducted a total of 24 60 -carrier UDM tests. The study director allowed an alternative approach in which a laboratory could conduct two 60 carrier tests/day, consisting of the current and modif ed methods for each high and low treatment. Under this scenario, an extra $24 \mathrm{~h}$ test microbe subculture was necessary to extend the use period of the test culture. Three untreated control carriers for each method were assayed each test day to measure the density of viable bacteria/carrier [expressed as the geometric mean colony forming units (CFUs)/carrier]. The evaluation of the control carriers was performed according to EPA SOP MB-05. An arithmetic mean minimum level of $6 \log _{10}$ (CFU/carrier), which corresponds to a geometric mean $\mathrm{CFU} /$ carrier minimum of $1 \times 10^{6}$, was expected for both test microbes. Testing was repeated if the minimum control carrier counts fell below the $6 \log _{10}(\mathrm{CFU} /$ carrier $)$ minimum.

\section{UDM Modif cations Evaluated in the Collaborative Study}

A single lyophilized ampule of each microbe was used by each laboratory to initiate two types of stocks (one for the current UDM and one for the modif ed UDM) on the same day. Brief y, for the current UDM, stocks of $S$. aureus were established and stored on nutrient agar slants; $P$. aeruginosa stocks were established and stored on cystine trypticase agar. Final test cultures for the current UDM were prepared in nutrient broth with a minimum of three consecutive $24 \mathrm{~h}$ transfers $(10 \mathrm{~mL}$ for daily transfers) followed by a f nal $48-54 \mathrm{~h} 20 \mathrm{~mL}$ test culture. For the modif ed UDM, frozen stocks and f nal test cultures were prepared as described below.

Preparation of frozen stock cultures for the modifed procedure.-Using a tube containing 5-6 $\mathrm{mL}$ of tryptic soy broth (TSB), aseptically withdraw 0.5 to $1.0 \mathrm{~mL}$ and rehydrate the lyophilized culture. Aseptically transfer the entire rehydrated pellet back into the original tube of broth. Mix well. Incubate for $24 \pm 2 \mathrm{~h}$ at $36 \pm 1^{\circ} \mathrm{C}$. Using a sterile spreader, inoculate a suff cient number of tryptic soy agar (TSA) plates (e.g., 5 to 10 plates/organism) with $100 \mu \mathrm{L}$ each of the culture. Incubate plates at $36 \pm 1{ }^{\circ} \mathrm{C}$ for $24 \pm 2 \mathrm{~h}$. Following incubation, add $5 \mathrm{~mL}$ cryoprotectant solution (TSB with $15 \% \mathrm{v} / \mathrm{v}$ glycerol) to the surface of each agar plate. Resuspend the cells in this solution using a sterile spreader or sterile swab, and aspirate 
the cell suspension from the surface of the agar. Transfer suspension into a sterile vessel. Repeat by adding another $5 \mathrm{~mL}$ of cryoprotectant to the agar plates, resuspend the cells, aspirate suspension, and pool with the initial cell suspension. Alternately, $10 \mathrm{~mL}$ of cryoprotectant solution may be added/plate for re-suspending with subsequent aspiration. Mix the pooled contents of the vessel thoroughly. Immediately after mixing, pipette approximately $1.0 \mathrm{~mL}$ of the diluted suspension into cryovials (e.g., $1.5 \mathrm{~mL}$ ). Place and store cryovials in $-70^{\circ} \mathrm{C}$ or below freezer.

Final test culture preparation for the modif ed procedure.Test culture preparation was consistent with AOAC Off cial Method 991.47. To prepare the f nal test cultures, defrost a single cryovial at room temperature and brief y vortex. Add $100 \mu \mathrm{L}$ of the thawed frozen stock to one $20 \times 150 \mathrm{~mm}$ tube containing $10 \mathrm{~mL}$ nutrient broth and incubate at $36 \pm 1^{\circ} \mathrm{C}$ for $24 \pm 2 \mathrm{~h}$. Using a sterile pipet, add $0.1 \mathrm{~mL}$ of $24 \pm 2 \mathrm{~h}$ culture to each of f ve TSA plates and spread culture onto plate to make a lawn. Incubate $24 \pm 2 \mathrm{~h}$ at $36 \pm 1^{\circ} \mathrm{C}$. To each plate add $10 \mathrm{~mL}$ nutrient broth. With sterile spreader or swab, gently swab surface of plate to remove growth. With sterile pipet, collect nutrient broth containing suspended bacterial cells, and pool in sterile glass f ask. Pass suspension, under vacuum, through $47 \mathrm{~mm}$ sterile absorbent pads using sterile f ltration unit. Use sterile absorbent pads with dispenser (47 mm diameter, cellulose fber pads; Millipore Corp., Billerica, MA). Collect the suspension in a sterile fask. Sonicate (ultrasonic cleaner) $5 \mathrm{~min}$ and let stand for $10 \mathrm{~min}$. Water level in sonicator must be at same level as suspension in f ask. Flask must contact bottom of sonicator. Use a timer for tracking time. Use the suspension as soon as possible and only on the day it is prepared. Refrigerate suspension when not in use. Using nutrient broth as the diluent, adjust the test suspension using optical density (OD) reading at $650 \mathrm{~nm}$ to a level comparable to the OD $( \pm 0.05)$ recorded for the f nal test culture for the current UDM. Use nutrient broth as a blank for assessing the OD. The adjusted suspension represents the f nal test culture for the modif ed method. Proceed with inoculation of carriers as indicated in the current UDM.

\section{Statistical Analysis}

\section{Data}

The data were peer-reviewed by an industry liaison and EPA and submitted for analysis as Excel fles for each laboratory. The worksheets were merged into two combined data matrixes, one for the control carrier data and one for the eff cacy data. The two data matrixes are displayed in Appendices 1 and 2.

The density of bacteria on each control carrier was recorded as CFU/carrier by the laboratory technician, and then $\log _{10}$-transformed to form the carrier log density [LD $=\log _{10}$ (CFU/carrier)]. The term Test $L D$ is used to denote the mean log density across the three carriers in a single test.

Three eff cacy response variables were analyzed: the number of positive carriers, the log reduction, and the pass/fail outcome. The traditional eff cacy response for the UDM is the number of positive carriers (denoted by $N P$ ) among the 60 treated carriers in a test. When interpreting this eff cacy response variable, it is important to remember that a high number of positive carriers indicates low disinfectant eff cacy.

Comparisons based only on the number of positive carriers are somewhat limited and f awed because they do not take into account the viable cell counts associated with the inoculated carriers. The log reduction (LR), the usual measure of eff cacy for quantitative disinfectant tests, accounts for any differences in the number of viable cells inoculated on the carriers in different tests. Consequently, being able to measure the eff cacy of the UDM in terms of LRs enables one to compare the variability of the UDM to literature values for other quantitative or semiquantitative disinfectant tests. This was the approach taken in an analysis of the UDM by Tomasino and Hamilton (11), and for the HSCT by Hamilton et al. (12). The LR for a single test is calculated by subtracting the mean log density for treated carriers from the mean log density for control carriers (19). For the UDM method, viable cell counts on the treated carriers are not available for directly calculating the mean log density for the treated carriers, but that mean can be estimated from the number of positive carriers out of 60 , which yields the following equation for the LR based on a modif ed most probable number formula (20),

$$
\mathrm{LR}=\operatorname{Test} L D-\log _{10}[-\ln ((60.5-N P) / 61)] .
$$

The pass/fail outcome is based on the performance standard for which a pass is recorded if there are 0 or 1 positive carriers out of 60 tested.

For each of the response variables (control LDs, number of positive carriers, LRs, pass/fail outcomes) considered in the collaborative study, diagnostic plots were used to detect the occurrence of outliers and inconsistent or unusual values. All such data were brought to the attention of the study director and subsequently validated; no data were rejected. Two data-related issues were noted: (1) in a few cases, a laboratory repeated a test because the control carrier counts were too low; and (2) the number of control carriers observed on each test day was sometimes three and sometimes six, but always the same number for both methods. The $\mathrm{f} \mathrm{rst}$ issue arose because the study protocol specif ed that the geometric mean density (CFU/carrier) must be at least $1 \times 10^{6}$. When the geometric mean density was below $1 \times 10^{6}$, the test was repeated. If a laboratory repeated a test for one method, it also repeated the test for the other method, even if the geometric mean density for the other method was at least $1 \times 10^{6}$. In one instance, a test was repeated a second time. For these reasons, the initial data set contained multiple results for some combinations of microbe, eff cacy level, replicate, and laboratory. After consultation with the study director, it was determined that if a test was repeated, only the data from the f nal repeat would be analyzed. Otherwise, all submitted data were included in the analysis. The second issue did not require a decision, but it created an unbalanced data set because some test days produced one set of three inoculated, untreated carrier counts, and other test days produced two sets of three carrier counts. This issue required that the statisticians $\mathrm{ft}$ the analysis of variance (ANOVA) models (described in the next section) using the method of restricted maximum likelihood (REML), which is suitable for unbalanced data (21).

\section{Method Performance Attributes (Resemblance, Responsiveness, Repeatability, and Reproducibility)}

The current and modif ed methods were evaluated separately to assess the extent to which each method achieved the desirable 
attributes of resemblance, responsiveness, repeatability, and reproducibility. Each of these four attributes is def ned next.

The inoculated carriers used in different tests and in different laboratories should resemble each other; that is, that the bacteria-laden carriers for the UDM tests should be similar from test to test. Resemblance was assessed by calculating standard deviations (SDs) for the control carrier log densities, both within and across laboratories. Small SDs indicate good resemblance of the untreated control carriers. The SDs were calculated by an ANOVA, which estimated four nested variance components: the variance among carriers within a test $\left(C S^{2}\right)$; the variance among replicate tests within the same test day $\left(C S_{\operatorname{Rep}(\operatorname{Day})}\right)^{2}$ ); the variance among multiple test days in a laboratory $\left(C S_{D a y}{ }^{2}\right)$; and the variance among laboratories $\left(\mathrm{CS}_{\mathrm{Lab}}{ }^{2}\right)$. The resemblance repeatability $\mathrm{SD}$

$$
C S_{r}=\sqrt{{C S^{2} / 3+C S_{\text {Rep(Day) }}^{2}+C S_{\text {Day }}^{2}}^{2}}
$$

is a measure of the within-laboratory variability of the Test $L D$ values. The resemblance reproducibility SD

$$
\mathrm{CS}_{\mathrm{R}}=\sqrt{\mathrm{CS}_{\mathrm{r}}^{2}+\mathrm{CS}_{\mathrm{Lab}}^{2}}
$$

is a measure of the total variability of the Test $L D$ values across all laboratories. Some guidance on the acceptable range of values for the repeatability $\mathrm{SD}\left(\mathrm{CS}_{\mathrm{r}}\right)$ and the reproducibility $\mathrm{SD}$ $\left(\mathrm{CS}_{\mathrm{R}}\right)$ for standardized disinfectant tests is provided by a review of historical data $(22,23)$. A value of $\mathrm{CS}_{\mathrm{r}}<0.5$ was found to be acceptable; $\mathrm{CS}_{\mathrm{R}}$ values $<0.7$ were found to be acceptable.

A method should be responsive to changes in disinfectant eff cacy. In this collaborative study, two presumably different eff cacy levels (low- and high-eff cacy) of the disinfectant were tested by both UDM methods on the same test day. That parallel testing allowed direct comparison of the test results for the low- and high-eff cacy levels. One way that responsiveness was measured was by the difference in the percentage of positive carriers between the two eff cacy levels on each test day

$$
\operatorname{RespNP}=\mathrm{P}_{\mathrm{L}}-\mathrm{P}_{\mathrm{H}}
$$

where the low-eff cacy percentage of positive carriers out of 60 is denoted by $\mathrm{P}_{\mathrm{L}}$, and the high eff cacy percentage of positive carriers out of 60 is $\mathrm{P}_{\mathrm{H}}$. Thus, the low- and high-eff cacy levels performed on the same day for the same microbe and method were paired. If RespNP is signif cantly larger than zero, then we conclude that the method is responsive. For each combination of method and microbe, a one-sided 95\% lower conf dence limit for the true mean responsiveness was calculated, along with the $P$-value for a one-sided test of the true responsiveness. The calculations are based on modif ed Price-Bonett binomial techniques (24) for linear combinations of binomial responses, which uses a normal approximation to the binomial distribution, an approximation that is justif ed for this study because each proportion is calculated from 60 carriers.

Responsiveness was also measured by the difference in LR values between the two eff cacy levels on each test day

$$
\operatorname{RespLR}=\mathrm{LR}_{\mathrm{H}}-\mathrm{LR}_{\mathrm{L}}
$$

where the low-eff cacy LR is denoted by $\mathrm{LR}_{\mathrm{L}}$, and the higheff cacy $L R$ is $L_{\mathrm{H}}$. For each combination of microbe and method, an ANOVA was $\mathrm{ft}$ to the RespLR values, with laboratory as a random effect. Based on the variance components estimated
Table 1. Statistical summary for control TestLD values for the current and modif ed use-dilution methods

\begin{tabular}{lcccc}
\hline Microbe & Method & Mean (SEM) & $\mathrm{CS}_{\mathrm{r}}$ & $\mathrm{CS}_{\mathrm{R}}$ \\
\hline P. aeruginosa & Current & $6.71(0.14)$ & 0.25 & 0.38 \\
P. aeruginosa & Modif ed & $6.68(0.09)$ & 0.36 & 0.37 \\
S. aureus & Current & $6.64(0.12)$ & 0.16 & 0.30 \\
S. aureus & Modif ed & $6.73(0.09)$ & 0.14 & 0.24 \\
\hline
\end{tabular}

a Averaged across laboratories, replicates, and tests for each combination of microbe and method.

from this ANOVA, a lower 95\% conf dence limit was calculated using a $t$-distribution with four degrees of freedom to ascertain whether the mean $\operatorname{RespLR}$ was statistically signif cantly greater than zero.

A disinfectant test method is deemed repeatable and reproducible if independent tests of the same treatment produce a similar eff cacy response. Repeatability pertains to independent tests in a single laboratory. Reproducibility pertains to independent tests in different laboratories. In this study, LR was the only eff cacy response used to assess repeatability and reproducibility since the LR response is directly comparable to results reported in the literature. The LR values for each combination of method, microbe, and eff cacy level, were analyzed by a one-way random effects ANOVA to estimate the repeatability (or within-laboratory) variance $S_{r}^{2}$ and the among-laboratory variance $\mathrm{S}_{\text {Lab }}^{2}(23)$. The repeatability $\mathrm{SD} \mathrm{S}_{\mathrm{r}}$ includes within-test variability and among-test variability for tests conducted in the same laboratory. The reproducibility $\mathrm{SD}, \mathrm{S}_{\mathrm{R}}=\left(\mathrm{S}_{\mathrm{r}}^{2}+\mathrm{S}_{\mathrm{Lab}}^{2}\right)^{1 / 2}$, includes among-laboratory variability, as well as the variability that makes up $\mathrm{S}_{\mathrm{r}}$. Note that $\mathrm{SD}$ is a measure of imprecision; therefore, small values of $S_{r}$ and $S_{R}$, typically calculated from collaborative studies of quantitative disinfectant tests, indicate good repeatability and reproducibility, respectively.

Some guidance on the acceptable range of $S_{r}$ and $S_{R}$ for standardized disinfectant tests is provided by a literature review (9). The review found that the LR values from accepted, standardized suspension and dried surface tests had $S_{R}$ that ranged from 0.3 to 1.5 , with a median of 0.9 , and $S_{r}$ that ranged from 0.2 to 1.2 , with a median of 0.5 . Typically, $50 \%$ of the total variance of $\mathrm{LR}$ values $\left(\mathrm{S}_{\mathrm{R}}{ }^{2}\right)$ was attributable to the variance among laboratories. More recently, Tomasino et al. (8) reported $\mathrm{S}_{\mathrm{r}}$ that ranged from 0.22 to 0.24 and $\mathrm{S}_{\mathrm{R}}$ that ranged from 0.29 to 0.32 for the UDM. These observations can be used as rough guidelines for evaluating the UDM test methods presented in this report.

Throughout the analyses, periodic checks were conducted

Table 2. The mean difference and $90 \%$ conf dence interval

\begin{tabular}{|c|c|c|c|c|c|}
\hline \multirow[b]{3}{*}{ Microbe } & \multirow{2}{*}{\multicolumn{2}{|c|}{ Mean TestLD }} & \multicolumn{3}{|c|}{ Test $L D$ difference: current-modif ed } \\
\hline & & & & $90 \%$ Conf c & nce interval \\
\hline & Current & Modif ed & Mean & Lower limit & Upper limit \\
\hline$P$. aeruginosa & 6.71 & 6.68 & 0.03 & -0.25 & 0.31 \\
\hline S. aureus & 6.64 & 6.73 & -0.08 & -0.36 & 0.19 \\
\hline
\end{tabular}
for the true mean difference between concurrently observed Test $L D$ values (current-modif ed), presented for each microbe 


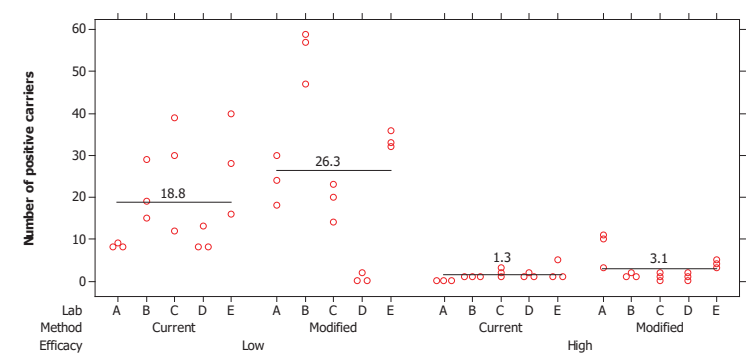

Figure 1. Number of positive carriers observed for $P$. aeruginosa. Each point is the result from a single test. The horizontal lines indicate the mean number of positive carriers for the low- and high-eff cacy levels for each of the two methods; the mean value is presented above the line.

to ensure that the data were not inadvertently altered during statistical manipulations. Normal probability and residual plots were used to determine that LDs and LRs satisf ed the normality and homogeneity of variance assumptions required by the ANOVA models. The ANOVA models were $\mathrm{ft}$ by REML using the nlme package in the software R (v. 2.11.0). All statements of statistical signif cance are made with respect to a signif cance level of $5 \%$.

\section{Method Comparison (Equivalence Testing)}

An important goal of the statistical analysis was to assess the equivalence of the two methods with respect to the following responses: the LDs of the untreated controls; the number of positive carriers; and the LRs. The assessment was based on statistical equivalence tests which determine if the observed differences between the two methods were negligibly small (11). In the equivalence tests, the following hypotheses were compared:

$$
\begin{aligned}
& \mathrm{H}_{0}:\left|\mu_{\text {current }}-\mu_{\text {modifed }}\right| \geq \delta \\
& \mathrm{H}_{\mathrm{a}}:\left|\mu_{\text {current }}-\mu_{\text {modifed }}\right|<\delta
\end{aligned}
$$

The parameter $\mu_{\text {modif ed }}$ is one of the true mean responses estimated in this study for the modif ed UDM (i.e., either the true mean control log density, the true percentage of positive carriers, or the true mean LR), and the parameter $\mu_{\text {current }}$ is the corresponding true mean UDM response for the current method. The equivalence parameter $\delta$ specif es the largest difference in

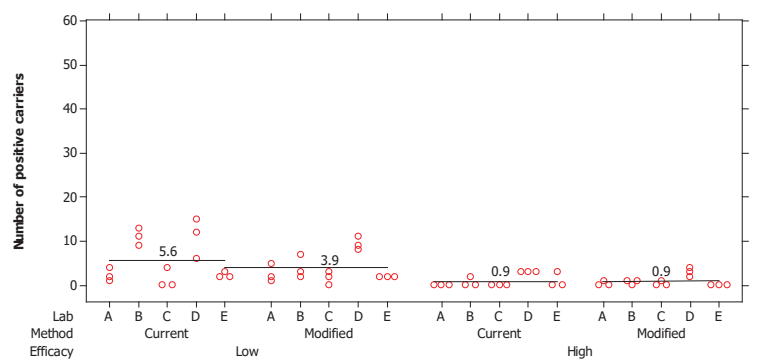

Figure 2. Number of positive carriers observed for $S$. aureus. Each point is the result from a single test. The horizontal lines indicate the mean number of positive carriers for the low- and high-eff cacy levels for each of the two methods; the mean value is presented above the line.

the responses that is negligible and not of practical importance. The null hypothesis $\mathrm{H}_{0}$ is rejected in favor of the hypothesis $\mathrm{H}_{\mathrm{a}}$ for equivalence of the two methods at a 5\% signif cance level when the 2 -sided $90 \%$ conf dence interval for $\mu_{\text {current }}-\mu_{\text {modifed }}$ is contained in $(-\delta, \delta)(25)$.

When comparing the true mean control log densities or the true mean LRs, the difference between the two methods was calculated for each test day; an ANOVA was then $\mathrm{ft}$ to these differences with laboratory as a random effect. Based on the variance components estimated from this ANOVA, a $90 \%$ conf dence interval for the true mean difference was calculated using a $t$-distribution with four degrees of freedom. When comparing the true percentage of positive carriers, the difference in percentages between the two methods was calculated for each test day; the Price-Bonett technique (24) was then applied to construct a $90 \%$ conf dence interval for the true difference.

For this UDM collaborative study, equivalence tests of the mean $\log$ densities of the untreated controls were conducted with $\delta=0.40$. Thus, absolute mean differences in the $\log$ densities of the viable organisms on the inoculated carriers as large as 0.40 on the $\log _{10}(\mathrm{CFU} /$ carrier) scale (which corresponds to a multiple of 2.5 on the $\mathrm{CFU} /$ carrier scale) were considered negligible and not of practical importance. Equivalence tests of the percentage of positive carriers were conducted with $\delta=5 \%$. Thus, absolute differences as large as $5 \%$ (i.e., differences up to three carriers) were considered negligible and not of practical importance. Equivalence tests of the LRs were conducted with $\delta=0.5$. Thus, absolute mean differences in the LRs as large as 0.5 were considered negligible and not of practical importance.

\begin{tabular}{|c|c|c|c|c|c|c|}
\hline \multirow[b]{3}{*}{ Microbe } & \multirow[b]{3}{*}{ Eff cacy level } & \multicolumn{2}{|c|}{ Percentage positive } & & & \\
\hline & & Current method & Modif ed method & \multicolumn{3}{|c|}{ Comparison of eff cacy: $\mathrm{P}_{\text {Cur }}-\mathrm{P}_{\text {Mod }}$} \\
\hline & & $P_{\text {Cur }} \%$ & $\mathrm{P}_{\text {Mod, }} \%$ & Mean, \% & $\begin{array}{c}90 \% 2 \text {-sided } \\
\text { Cl lower limit, } \%^{a}\end{array}$ & $\begin{array}{c}90 \% \text { 2-sided } \\
\mathrm{Cl} \text { upper limit, } \%^{a}\end{array}$ \\
\hline$P$. aeruginosa & High & 2.2 & 5.1 & -2.9 & -4.3 & -1.5 \\
\hline$P$. aeruginosa & Low & 31.3 & 43.9 & -12.6 & -15.7 & -9.4 \\
\hline S. aureus & High & 1.6 & 1.4 & 0.2 & -0.8 & 1.1 \\
\hline S. aureus & Low & 9.3 & 6.6 & 2.8 & 0.7 & 4.8 \\
\hline
\end{tabular}

Table 3. Comparison of eff cacy based on the number of positive carriers

a Calculated using the Price-Bonett technique. 


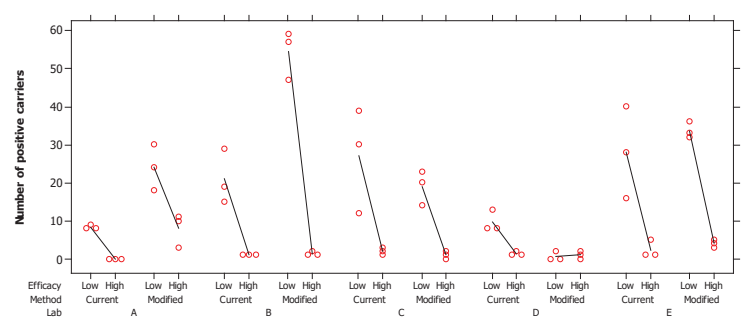

Figure 3. Assessment of method responsiveness for $P$. aeruginosa where each point represents the number of positive carriers from a single test/method. The lines connect the low- and high-eff cacy levels for each method.

\section{Results and Discussion}

\section{Comparison of the Untreated Control Carrier Counts (TestLD Values)}

The control Test $L D$ values reported in this study and the repeatability control $\mathrm{CS}_{\mathrm{r}}$ and reproducibility control $\mathrm{CS}_{\mathrm{R}}$ values were very similar to those previously reported (8). The mean control Test $L D$ values, variance components, and $\mathrm{CS}_{\mathrm{r}}$ and $\mathrm{CS}_{\mathrm{R}}$ SDs for each combination of microbe and method are displayed in Table 1. Two of the Test $L D$ values for the modif ed method marginally fell below 6.0 and were repeated and not included in the analysis; otherwise, all other control TestLDs were above the prescribed minimum of 6.0. The mean LDs are almost identical for each combination of microbe and method ranging from 6.64 to 6.73 . The use of OD readings to adjust the f nal test culture preparations for the modif ed UDM was a valuable tool to ensure that the carrier counts were comparable between the two methods. Thus, the similar Test $L D s$ strengthened our ability to compare the performance of the two methods.

Based on the $\mathrm{CS}_{\mathrm{r}}$ and $\mathrm{CS}_{\mathrm{R}} \mathrm{SDs}$, the control TestLDs for $P$. aeruginosa were slightly more variable than those for $S$. aureus; however, both methods exhibited acceptably small $\mathrm{S}_{\mathrm{r}}$ (range of 0.14 to 0.36 ) and $\mathrm{S}_{\mathrm{R}}$ (range of 0.24 to 0.38 ). The statistical analysis of the differences between control Test $L D$ values for the two methods, current minus modif ed, for each pair of control carrier counts is presented in Table 2. The table shows that the conf dence limit farthest from zero is -0.36 . Thus, as long as mean $\log _{10}(\mathrm{CFU} /$ carrier) differences as large as 0.40 are considered negligible, then the Test $L D$ values for the current and modif ed methods are statistically signif cantly equivalent. Therefore, although different procedures were used for the

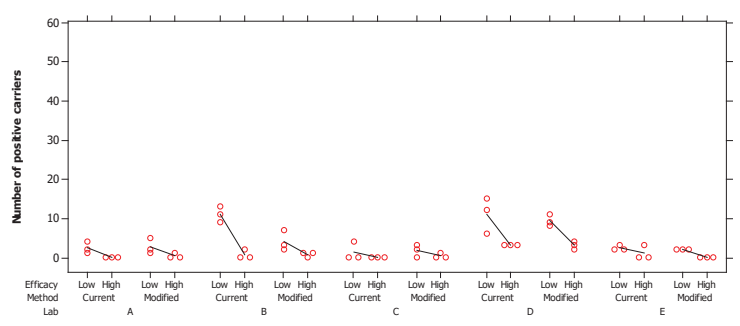

Figure 4. Assessment of method responsiveness for $S$. aureus where each point represents the number of positive carriers from a single test/method. The lines connect the means at low- and high-eff cacy levels for each method.

preparation of the f nal test cultures, both methods produced acceptable and statistically equivalent control TestLDs.

\section{Comparison of Product Eff cacy Results Based on the Number of Positive Carriers}

For each method, the numbers of positive carriers/test for each laboratory are plotted in Figure 1 for $P$. aeruginosa and in Figure 2 for $S$. aureus. Across eff cacy levels and methods, the number of positive carriers observed for $S$. aureus was lower than those for $P$. aeruginosa. Across microbes and methods, the highest degree of variability was noted at the low-eff cacy level. Laboratory D generated a low number of positive carriers for the modif ed method at the low-eff cacy treatment for $P$. aeruginosa; however, based on a discussion with the laboratory staff and an inspection of the raw data, the data from laboratory D were considered valid and used in the analysis. The number of positive carriers across both methods was far less variable for the high-eff cacy treatment. The product was more dilute for the low eff cacy treatment for $S$. aureus (1:1280) compared to that for P. aeruginosa (1:576); thus, our ability to compare the differences in product eff cacy between microbes is limited at the low-eff cacy level.

For $P$. aeruginosa, the high eff cacy treatment yielded a similar number of positives (mean of 1.3 positives out of 60 for the current method and 3.1 positives out of 60 for the modif ed method). However, the overall variability in the results was greater for the modif ed method: range of 0 to 5 positives for the current method, compared to 0 to 11 for the modif ed method. Both methods produced a mean of 0.9 positives out of

Table 4. Responsiveness based on the number of positive carriers

\begin{tabular}{|c|c|c|c|c|c|c|}
\hline \multirow[b]{3}{*}{ Microbe } & \multirow[b]{3}{*}{ Method } & \multicolumn{2}{|c|}{ Percentage positive } & \multicolumn{3}{|c|}{ Responsiveness: RespNP $=\mathrm{P}_{\mathrm{L}}-\mathrm{P}_{\mathrm{H}}$} \\
\hline & & Low-eff cacy & High-eff cacy & & & \\
\hline & & $P_{L}$ & $\mathrm{P}_{\mathrm{H}}$ & Mean & $95 \%$ one-sided $\mathrm{Cl}$ (lower limit) ${ }^{a}$ & $P$-value, one-tailed ${ }^{a}$ \\
\hline$P$. aeruginosa & Current & $31.3 \%$ & $2.2 \%$ & $29.1 \%$ & $26.6 \%$ & $<0.001$ \\
\hline$P$. aeruginosa & Modif ed & $43.9 \%$ & $5.1 \%$ & $38.8 \%$ & $36.3 \%$ & $<0.001$ \\
\hline S. aureus & Current & $9.3 \%$ & $1.6 \%$ & $7.8 \%$ & $6.1 \%$ & $<0.001$ \\
\hline S. aureus & Modif ed & $6.6 \%$ & $1.4 \%$ & $5.1 \%$ & $3.6 \%$ & $<0.001$ \\
\hline
\end{tabular}

a Calculated using the Price-Bonett technique. 


\begin{tabular}{|c|c|c|c|c|c|c|}
\hline \multirow[b]{3}{*}{ Microbe } & \multirow[b]{3}{*}{ Eff cacy level } & & & \multicolumn{3}{|c|}{ LR Difference: current-modif ed } \\
\hline & & \multicolumn{2}{|c|}{ Mean LR } & \multirow[b]{2}{*}{ Mean } & \multicolumn{2}{|c|}{$90 \%$ Conf dence interval } \\
\hline & & Current & Modif ed & & Lower limit & Upper limit \\
\hline$\overline{P . \text { aeruginosa }}$ & Low & 7.25 & 7.11 & 0.15 & -0.60 & 0.90 \\
\hline$P$. aeruginosa & High & 8.26 & 8.09 & 0.17 & -0.35 & 0.70 \\
\hline S. aureus & Low & 7.79 & 8.00 & -0.21 & -0.48 & 0.07 \\
\hline
\end{tabular}

60 positives for $S$. aureus for the high treatment with a range of 0-4 positive carriers across the methods.

For $P$. aeruginosa, the mean number of positive carriers for the low-eff cacy treatment was 18.8 and 26.3 for the current and modif ed method, respectively. For the low treatment, the range in positives for $P$. aeruginosa was greater: 9-40 and 0-55 for the current and modif ed methods, respectively. For $S$. aureus, the current method yielded a mean of 5.6 positives for the low eff cacy treatment. Similarly, a mean of 3.9 positives was observed for the low-eff cacy treatment for the modif ed method.

Across all combinations of method, microbe, and eff cacy level, the mean percentages of positive carriers out of 60 for the current method $\left(\mathrm{P}_{\mathrm{Cur}}\right)$, and the modif ed method $\left(\mathrm{P}_{\mathrm{Mod}}\right)$ are presented and compared $\left(\mathrm{P}_{\mathrm{Cur}}-\mathrm{P}_{\mathrm{Mod}}\right)$ in Table 3. The absolute difference in numbers of positive carriers between methods was greatest for the $P$. aeruginosa/low eff cacy combination (12.6\% difference, corresponding to 7.6 positives out of 60 carriers). The wide conf dence interval indicates a failure to ascertain statistical equivalence of the two methods for the P. aeruginosal low eff cacy combination. The percentage of positive carriers was very similar across methods for $S$. aureus; an equivalence test shows that the methods produce statistically signif cantly equivalent percentages of positive carriers for both low- and high-eff cacy levels for $S$. aureus, as long as percentages as large as $4.8 \%$ (equivalent to $2.9 / 60$ carriers) are considered small and negligible.

These results suggest that the $P$. aeruginosa inoculum generated using the agar-based approach is more tolerant of disinfection than the current broth-based approach and produces more variable results. However, further studies would be necessary to fully characterize the differences. Alternatively, it is possible that the modif ed method as written is more challenging to perform and additional practice runs are necessary to optimize the results. The use of frozen stock cultures is a routine practice used by microbiology laboratories that conduct standardized test methods, and should not have inf uenced the outcome of the modif ed approach. However, the harvesting and standardization of inoculum using the agar-based approach involves several steps and may be responsible for the increase in variability.

\section{Assessment of Method Responsiveness Based on the Number of Positive Carriers}

To assess method responsiveness, the numbers of positive carriers for each microbe and method were plotted to compare the low- and high-eff cacy data side-by-side (Figures 3 and 4). The data are further summarized as percentage of positives with the associated statistics in Table 4. With the exception of laboratory D for P. aeruginosa, both methods exhibited the ability to detect the presumed eff cacy-response relationship. A statistical analysis showed that the percentages of positive carriers for all combinations of method and microbe are signif cantly lower $(P<0.001)$ for the high-eff cacy treatment (the mean differences ranged from $5.1 \%$ to $38.8 \%$ ) compared to the low-eff cacy treatment. Thus, both versions of the UDM are statistically signif cantly responsive to the two eff cacy levels of the product.

\section{Comparison of Product Eff cacy Based on LR}

The mean $L R$ values for each microbe and method combination are provided in Table 5. The LRs reinforce conclusions based on differences in product eff cacy results using the number of positive carriers. Across the four combinations of microbe and eff cacy level, the mean LR values ranged from 7.1 to 8.5. Absolute mean differences in the LR between the current and modif ed methods across microbes and eff cacy levels were small, ranging from 0.01 to 0.21 . Since the conf dence intervals were wide for tests with $P$. aeruginosa ( 0.9 low- eff cacy upper conf dence limit and a 0.7 high-eff cacy upper conf dence limit),

Table 6. Repeatability SD and reproducibility SD of LR for each of the two methods, for each combination of microbe and eff cacy level

\begin{tabular}{|c|c|c|c|c|c|}
\hline \multirow[b]{2}{*}{ Microbe } & \multirow[b]{2}{*}{ Efficacy level } & \multicolumn{2}{|c|}{$\mathrm{S}_{\mathrm{r}}$} & \multicolumn{2}{|c|}{$S_{R}$} \\
\hline & & Current & Modified & Current & Modified \\
\hline$P$. aeruginosa & Low & 0.15 & 0.42 & 0.55 & 1.10 \\
\hline$P$. aeruginosa & High & 0.33 & 0.50 & 0.53 & 0.53 \\
\hline S. aureus & Low & 0.26 & 0.34 & 0.36 & 0.40 \\
\hline S. aureus & High & 0.32 & 0.32 & 0.32 & 0.39 \\
\hline
\end{tabular}



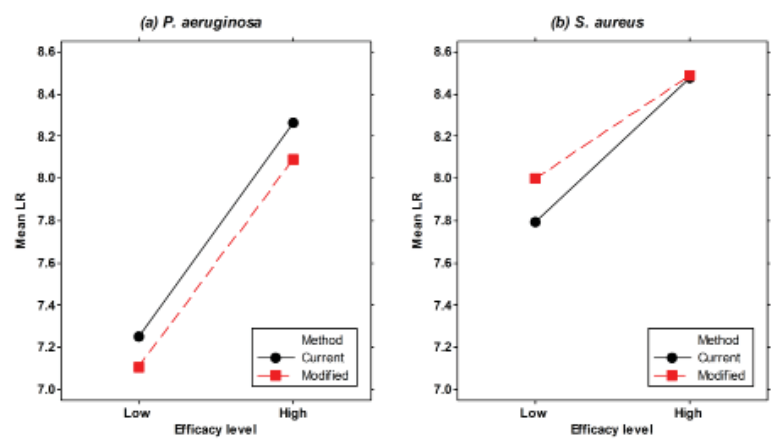

Figure 5. Comparison of responsiveness using the LR. Each point is the mean LR across 15 tests (three replications in each of $f$ ve laboratories). The lines connect the low- and high-eff cacy levels for each method:

(a) $P$. aeruginosa tests and (b) $S$. aureus tests.

the collaborative study data fail to support equivalency of the LR values for the current and modif ed methods applied to $P$. aeruginosa. For tests with $S$. aureus, the narrow conf dence intervals show that the mean LR values for the current and modif ed methods are statistically signif cantly equivalent as long as absolute mean LR differences as large as 0.5 are considered small and negligible.

The $S_{r}$ and $S_{R}$ SDs for the LR are provided in Table 6. For each combination of microbe and eff cacy level, both methods produced acceptably small $S_{r}$ and $S_{R}$ values (10). Overall, $S_{r}$ and $\mathrm{S}_{\mathrm{R}}$ were less for $S$. aureus tests than for the corresponding $P$. aeruginosa tests. Across the combinations of microbe and eff cacy level, the $S_{r}$ values ranged from 0.15 to 0.33 for the current method and from 0.32 to 0.50 for the modif ed method; the $S_{R}$ values ranged from 0.32 to 0.55 for the current method and from 0.39 to 1.10 for the modif ed method. Based on the $S_{r}$ and $S_{R}$ values for the LR, the modif cations did not reduce method variability.

\section{Comparison of Responsiveness Based on Assessment of $L R$}

To assess and compare overall method responsiveness with respect to the LRs, the mean LR values across the 15 replicate tests per microbe are plotted in Figure 5. The LR data reinforce conclusions reached on responsiveness using the number of positive carriers. For each microbe, the slopes for both methods are very similar. For $S$. aureus, the low-eff cacy level produced uniformly large LR values, thereby causing smaller responsiveness measures than for the corresponding $P$. aeruginosa tests. Table 7 presents a statistical summary of the responsiveness measure, including the mean and a $95 \%$ one-sided conf dence interval for the true mean. For each combination of microbe and method, the lower conf dence limit is positive and the $P$-value is very small. Thus, the mean LR at the high-eff cacy level is statistically signif cantly larger than the mean LR at the low-eff cacy level $(P \leq 0.021)$. This conf rms that both methods are highly responsive to changes in product eff cacy.

\section{Assessment of the Pass/Fail Outcome for the Product}

The current performance standard (which def nes a passing outcome) established for the UDM and accepted by the EPA for liquid disinfectants is 0 to 1 positive carriers out of 60 tested. In this study, the high eff cacy treatment conditions were based on the label claim of the product; thus, the high-eff cacy treatment was expected to pass the UDM and was deemed most relevant to this evaluation. The pass/fail outcome for the higheff cacy treatment is summarized in Table 8 for $S$. aureus and Table 9 for $P$. aeruginosa. For the $S$. aureus and high eff cacy treatment combination, $10 / 15(67 \%)$ and $12 / 15(80 \%)$ tests passed for the current and modif ed methods, respectively. For $P$. aeruginosa and the high-eff cacy treatment combination, $11 / 15(73 \%)$ tests with the current method passed while only $6 / 15(40 \%)$ tests passed for the modif ed counterpart. For the low-eff cacy treatment, only $3 / 15$ and $2 / 15$ tests passed the current and modif ed method, respectively, for $S$. aureus. For the $P$. aeruginosa and low-eff cacy treatment combination, the current method passed $0 / 15$ times, while $4 / 15$ tests passed the modif ed method. These data indicate that the modif cations did not improve method performance. Thus, the modif cations tested in this study, except the use of frozen stock cultures, will not be pursued as off cial procedural changes.

\section{Conclusions}

Overall, the targeted modif cations did not improve the performance of the UDM, i.e., the $S_{r}$ and $S_{R}$ for the modif ed method were equal to or greater than the $S_{r}$ and $S_{R}$ for the current method across the various test variables. In fact, it appears that the modif cations increased the variability in the results. The control Test $L D$ s consistently met the $6 \log _{10}(\mathrm{CFU} /$ carrier) minimum and were comparable for both methods. The values for $\mathrm{CS}_{\mathrm{r}}$ and $\mathrm{CS}_{\mathrm{R}}$ SDs for the TestLDs were acceptably small $(21,22)$, and were similar to data previously published (8).

Table 7. The mean responsiveness measure $R \operatorname{esp} L R=L R_{H}-L R_{L}$ for $L R$ values, the $95 \%$ lower, one-sided conf dence limit for the true mean $R \operatorname{esp} L R$, and the one-tailed $P$-value, presented for each combination of microbe and method

\begin{tabular}{|c|c|c|c|c|c|c|}
\hline \multirow[b]{3}{*}{ Microbe } & \multirow[b]{3}{*}{ Method } & \multirow{2}{*}{\multicolumn{2}{|c|}{ Mean LR }} & \multicolumn{3}{|c|}{ Responsiveness: RespLR } \\
\hline & & & & \multirow[b]{2}{*}{ Mean } & \multicolumn{2}{|c|}{$95 \%$ Conf dence interval } \\
\hline & & High-eff cacy & Low-eff cacy & & Lower limit (one-sided) & $\begin{array}{l}P \text {-value (one- } \\
\text { tailed) }\end{array}$ \\
\hline$P$. aeruginosa & Current & 8.26 & 7.25 & 1.01 & 0.80 & $<0.001$ \\
\hline$P$. aeruginosa & Modif ed & 8.09 & 7.11 & 0.98 & 0.27 & 0.021 \\
\hline S. aureus & Current & 8.48 & 7.79 & 0.68 & 0.42 & 0.003 \\
\hline S. aureus & Modif ed & 8.49 & 8.00 & 0.49 & 0.31 & 0.002 \\
\hline
\end{tabular}


Table 8. Number of positive carriers and Pass/Fail outcome for the $S$. aureus/high QAC eff cacy treatment for current and modif ed UDM

\begin{tabular}{|c|c|c|c|}
\hline \multirow[b]{2}{*}{ Laboratory } & \multirow[b]{2}{*}{ Replication } & \multicolumn{2}{|c|}{ No. positives out of 60 carriers } \\
\hline & & Current method $^{a}$ & Modif ed method $^{a}$ \\
\hline \multirow[t]{3}{*}{ A } & 1 & 0 (Pass) & 0 (Pass) \\
\hline & 2 & 0 (Pass) & 1 (Pass) \\
\hline & 3 & 0 (Pass) & 0 (Pass) \\
\hline \multirow[t]{3}{*}{ B } & 1 & 0 (Pass) & 0 (Pass) \\
\hline & 2 & 0 (Pass) & 1 (Pass) \\
\hline & 3 & 2 (Fail) & 1 (Pass) \\
\hline \multirow[t]{3}{*}{ C } & 1 & 0 (Pass) & 0 (Pass) \\
\hline & 2 & 0 (Pass) & 1 (Pass) \\
\hline & 3 & 0 (Pass) & 0 (Pass) \\
\hline \multirow[t]{3}{*}{ D } & 1 & 3 (Fail) & 2 (Fail) \\
\hline & 2 & 3 (Fail) & 3 (Fail) \\
\hline & 3 & 3 (Fail) & 4 (Fail) \\
\hline \multirow[t]{4}{*}{ E } & 1 & 0 (Pass) & 0 (Pass) \\
\hline & 2 & 0 (Pass) & 0 (Pass) \\
\hline & 3 & 3 (Fail) & 0 (Pass) \\
\hline & & $10 / 15$ Pass & 12/15 Pass \\
\hline
\end{tabular}

Table 9. Number of positive carriers and Pass/Fail outcome for the $P$. aeruginosa/high QAC eff cacy treatment for current and modif ed UDM

\begin{tabular}{|c|c|c|c|}
\hline \multirow[b]{2}{*}{ Laboratory } & \multirow[b]{2}{*}{ Replication } & \multicolumn{2}{|c|}{ No. positives out of 60 carriers } \\
\hline & & Current method $^{a}$ & Modif ed method \\
\hline \multirow[t]{3}{*}{ A } & 1 & 0 (Pass) & 10 (Fail) \\
\hline & 2 & 0 (Pass) & 11(Fail) \\
\hline & 3 & 0 (Pass) & 3 (Fail) \\
\hline \multirow[t]{3}{*}{ B } & 1 & 1 (Pass) & 1 (Pass) \\
\hline & 2 & 1 (Pass) & 2 (Fail) \\
\hline & 3 & 1 (Pass) & 1 (Pass) \\
\hline \multirow[t]{3}{*}{ C } & 1 & 3 (Fail) & 1 (Pass) \\
\hline & 2 & 2 (Fail) & 2 (Fail) \\
\hline & 3 & 1 (Pass) & 0 (Pass) \\
\hline \multirow[t]{3}{*}{$\mathrm{D}$} & 1 & 1 (Pass) & 0 (Pass) \\
\hline & 2 & 2 (Fail) & 2 (Fail) \\
\hline & 3 & 1 (Pass) & 1 (Pass) \\
\hline \multirow[t]{4}{*}{$E$} & 1 & 5 (Fail) & 4 (Fail) \\
\hline & 2 & 1 (Pass) & 5 (Fail) \\
\hline & 3 & 1 (Pass) & 3 (Fail) \\
\hline & & 11/15 Pass & 6/15 Pass \\
\hline
\end{tabular}

a Pass/Fail outcome: 0 to 1 positive out of 60 is a Pass; 2 and above positives out of 60 is a Fail.
Furthermore, based on differences between the number of positive carriers and the LR values between high- and loweff cacy treatments, both methods were deemed responsive to presumed changes in eff cacy. The modif ed method with $P$. aeruginosa generated a $12.6 \%$ (7.6 carriers) increase in the number of positive carriers for the low-eff cacy treatment compared to the current method, thus not supporting equivalency. The pass/fail data suggest an increased likelihood of failing an effective product when utilizing the modif ed method for $P$. aeruginosa. A greater range in the number of positives for $P$. aeruginosa was observed for the modif ed method (0-11 carriers) compared to the current method (0-5 carriers) for the high-eff cacy treatment. The corresponding LR values for $P$. aeruginosa also show a decrease in the mean LR for the modif ed method. The difference in positive carriers for $S$. aureus across methods was low and was $0.2 \%$ ( 0 carriers out of 60 ) for the high treatment and 2.8\% (1.7 carriers out of $60)$ for the low treatment. Thus, the modif cations impacted $S$. aureus much less than $P$. aeruginosa.

The current UDM, as performed in this study, displayed acceptable responsiveness to changes in product eff cacy: acceptable repeatability across multiple tests in each laboratory for the control LDs and the LRs; and acceptable reproducibility across multiple laboratories for the control LDs and the LRs. However, using the pass/fail criterion, one might argue that the method's variability remains too high for reliable use as a regulatory method with the current performance standard of $0-1$ positive out of 60 , especially for $P$. aeruginosa. These conclusions assume that the product tested is formulated to exhibit a predictable outcome (i.e., results for a "gold" standard product) under repeated testing across several laboratories; however, this conclusion cannot be made without further analysis. Based on the data and the associated statistical analysis provided in this report, a reassessment of the performance standard is being pursued by the EPA.

It is clear that conducting the current UDM using the same standardized procedure with trained analysts will provide more consistent results. Although there may be advantages (e.g., standardized inoculum) for the use of the modif ed method to generate f nal test cultures (i.e., reduce potential impact of the $P$. aeruginosa pellicle on method variability), the data reported here do not support its adoption into the UDM. Thus, pursuing the modif cations, with the exception of the frozen stocks, is not recommended. The HSCT approach generates a test suspension that is highly ref ned and may signif cantly change the quality of the inoculum and/or the physiological state of the test bacteria. These changes may result in differences in bacterial attachment and distribution on the carrier surface, and may be responsible for changes in tolerance to disinfectants, which was most evident in the P. aeruginosa data. Further experimentation is required to conf rm this theory. However, the authors propose retaining the frozen stock preparation step of the modif ed method, and based on the statistical equivalency of the control log densities, support its adoption as a procedural change to the current UDM. Furthermore, the use of frozen stock cultures is a widespread practice in microbiology laboratories and will reduce the likelihood of genetic drift due to frequent subculturing of the test bacteria. The proposed revision would eliminate the use of stock cultures grown and stored on nutrient agar slants (955.15) and cystine tryptic agar stabs (964.02), and is provided below. 


\section{Reagents}

Preparation of frozen stock cultures for the modifed procedure.-Using a tube containing 5-6 $\mathrm{mL}$ of TSB, aseptically withdraw $0.5-1.0 \mathrm{~mL}$ and rehydrate the lyophilized culture. Aseptically transfer the entire rehydrated pellet back into the original tube of broth. Mix well. Incubate for $24 \pm 2 \mathrm{~h}$ at $36 \pm 1^{\circ} \mathrm{C}$. Using a sterile spreader, inoculate a suff cient number of TSA plates (e.g., 5-10 plates/organism) with $100 \mu \mathrm{L}$ each of the culture. Incubate plates at $36 \pm 1{ }^{\circ} \mathrm{C}$ for $24 \pm 2 \mathrm{~h}$. Following incubation, add $5 \mathrm{~mL}$ cryoprotectant solution (TSB with $15 \%$ $\mathrm{v} / \mathrm{v}$ glycerol) to the surface of each agar plate. Resuspend the cells in this solution using a sterile spreader or a sterile swab, and aspirate the cell suspension from the surface of the agar. Transfer the suspension into a sterile vessel. Repeat by adding another $5 \mathrm{~mL}$ cryoprotectant to the agar plates, resuspend the cells, aspirate suspension, and pool with the initial cell suspension.

Alternately, $10 \mathrm{~mL}$ cryoprotectant solution may be added/ plate for resuspending with subsequent aspiration. Mix the pooled contents of the vessel thoroughly. Immediately after mixing, pipette approximately $1.0 \mathrm{~mL}$ of the diluted suspension into cryovials (e.g., $1.5 \mathrm{~mL}$ ). Place and store cryovials in $-70^{\circ} \mathrm{C}$ or below freezer. Store these frozen stock cultures up to 18 months.

\section{Acknowledgments}

The research that led to the preparation of this article was a collaborative effort between the EPA Off ce of Pesticide Programs Biological and Economic Analysis Division and the company members of the Eff cacy Working Group, including The Clorox Company, Ecolab, Inc., Lonza, Inc., Mason Chemical Company, Reckitt Benckiser, S.C. Johnson, and Stepan Company. These companies provided technical input, test material, and laboratory participation in the research. The statistical analyses were performed under contract between the Center for Biof $1 \mathrm{~m}$ Engineering at Montana State University and the EPA Off ce of Pesticide Programs, Biological and Economic Analysis Division, Microbiology Laboratory Branch. We thank Kiran Verma, Quality Assurance Off cer for the EPA Microbiology Laboratory, for conducting the audit of data that appear in this report.

\section{References}

(1) Off cial Methods of Analysis (2009) 18th Ed., AOAC INTERNATIONAL, Gaithersburg, MD, Method 955.14

(2) Off cial Methods of Analysis (2009) 18th Ed., AOAC INTERNATIONAL, Gaithersburg, MD, Method 955.15

(3) Off cial Methods of Analysis (2009) 18th Ed., AOAC INTERNATIONAL, Gaithersburg, MD, Method 964.02

(4) Cole, E.C., \& Rutala, W.A. (1986) J. AOAC Int. 69, 1003-1005

(5) Cole, E.C., Rutala, W.A., \& Carson, J.L. (1987) J. AOAC Int. 70, 903-909
(6) Off cial Methods of Analysis (2009) 18th Ed., AOAC INTERNATIONAL, Gaithersburg, MD, Method 991.47

(7) Rubino, R.R., Bauer, J.M., Clarke P.H., Woodward, B.B., Porter, F.C., \& Hilton, H.G. (1992) J. AOAC Int. 75, 635-645

(8) Tomasino, S.F., Pines, R.M., \& Hamilton, M.A. (2009) J. AOAC Int. 92, 1531-1540

(9) Tomasino, S.F., Pines, R.M., \& Hamilton, G.C. (2012) J. AOAC Int. 95, 1059-1063

(10) Tilt, N., \& Hamilton, M.A. (1999) J. AOAC Int. 82, 384-389

(11) Tomasino, S.F., \& Hamilton, M.A. (2006) J. AOAC Int. 89, 1373-1397

(12) Hamilton, M., Devries, T., \& Rubino, J. (1995) J. AOAC Int. 78 , 1102-1109

(13) ASTM International (2002) E 2197-02: Standard Quantitative Disk Carrier Test Method for Determining the Bactericidal, Virucidal, Fungicidal, Mycobactericidal and Sporicidal Activities of Liquid Chemical Germicides, West Conshohocken, PA

(14) Alfano, E.M., Cole, E.C., \& Rutala, W.A. (1988) J. AOAC Int. 71, 868-871

(15) Arlea, C., King, S., Bennie, B., Kemp, K., Mertz, E., \& Staub, R. (2008) J. AOAC Int. 91, 152-158

(16) U.S. Environmental Protection Agency (2010) Standard Operating Procedure (MB-05) for Conducting the Use-Dilution Method, Washington, DC

(17) Code of Federal Regulations (2005) Part 160-Good Laboratory Practice Standards, U.S. Government Printing Off ce, Washington, DC

(18) Off cial Methods of Analysis (2012) 18th Ed., AOAC INTERNATIONAL, Gaithersburg, MD, Method 960.09

(19) Zelver, N., Hamilton, M., Goeres, D., \& Heersink, J. (2001) Methods in Enzymology-Biof lms II 337, 363-376

(20) Hamilton, M. (2011) The P/N formula for the log reduction when using a semi-quantitative disinfectant test of type SQ1, KSA-SM-08, 2011-02-01, Center for Biof lm Engineering, Montana State University, Bozeman, MT, http://www.biof lm. montana.edu/resources/ knowledge sharing articles

(21) Pinheiro, C., \& Bates, D. (2000) Mixed-Effects Models in $S$ and $S$-PLUS, Statistics and Computing Series, Springer-Verlag, New York, NY. http://dx.doi.org/10.1007/978-1-4419-0318-1

(22) Hamilton, M.A. (2010) Testing Surface Disinfectants: Desirable Attributes of a Standardized Method, KSA-SM-03, 2010-0610, Center for Biof $1 \mathrm{~m}$ Engineering, Montana State University, Bozeman, MT. http://www.biof lm.montana.edu/ resources/ knowledge_sharing_articles

(23) Parker, A.E., \& Hamilton, M.A. (2011) Assessing Resemblance, Repeatability, and Reproducibility for Quantitative Methods, KSA-SM-10, 2011-06-23, Center for Biof Im Engineering, Montana State University, Bozeman, MT. http://www.biof lm. montana.edu/resources/knowledge_sharing_articles

(24) Price, R.M., \& Bonett, D.G. (2004) Comput. Stat. Data Anal. 45, 449-456. http://dx.doi.org/10.1016/S0167-9473(03)00007-0

(25) Richter, S.J., \& Richter, C. (2002) Quality Engineering 14, 375-380. http://dx.doi.org/10.1081/QEN-120001876 